\title{
Temporal variability in embryonic development and mortality in the southern calamary Sepioteuthis australis: a field assessment
}

\author{
M. A. Steer ${ }^{1,2, *}$, N. A. Moltschaniwskyj ${ }^{1}$, F. C. Gowland ${ }^{3}$ \\ ${ }^{1}$ School of Aquaculture, Tasman Aquaculture and Fisheries Institute, University of Tasmania, Locked Bag 1-370, Launceston, \\ Tasmania 7520, Australia \\ ${ }^{2}$ Marine Research Laboratories, Tasmanian Aquaculture and Fisheries Institute, University of Tasmania, PO Box 252-49, \\ Hobart, Tasmania 7001, Australia \\ ${ }^{3}$ Department of Zoology, University of Aberdeen, Aberdeen AB9 2TN, Scotland, UK
}

\begin{abstract}
This study describes the incidence of embryonic mortality and differential development in southern calamary Sepioteuthis australis eggs. Late-stage $S$. australis egg strands harbouring multiple embryos close to hatching were sampled from shallow $(<4 \mathrm{~m})$ Tasmanian spawning grounds from early November 2000 to January 2001. Sepioteuthis australis embryos were found to develop asynchronously within individual egg strands with proximal embryos developing slower and suffering higher mortality than their distal siblings. The magnitude of asynchrony, however, differed throughout the season with greater within-strand differences observed when embryos were exposed to broader incubation temperatures. Unexpectedly, embryos developed more synchronously within biologically fouled strands and displayed a significantly lower incidence of mortality compared to those developing in unfouled strands. Embryonic mortality was initially low (4\%) and significantly increased to $20 \%$ in late November, remaining above $10 \%$ until late December. This dramatic increase in mortality was not strongly associated with increasing water temperatures, but coincided with a period of heavy rainfall alluding to potential salinity effects.
\end{abstract}

KEY WORDS: Squid $\cdot$ Embryonic development $\cdot$ Mortality $\cdot$ Egg position $\cdot$ Biofouling $\cdot$ Asynchrony

\section{INTRODUCTION}

Quantitative estimates of mortality rates for squid species are virtually non-existent. However, they have been vaguely defined as higher than mammals, similar to marine plankton and lower than fish (O'Dor 1998). This description arises purely as a function of the logistical and technical complexities associated with reliably sampling the squid species through the egg, paralarval and juvenile phases in the wild. Nevertheless, the need to quantify early mortality rates and identify processes responsible is important, especially from a fisheries management perspective, as it can potentially be used to predict recruitment strength.

\footnotetext{
*Email: masteer@utas.edu.au
}

For most fish species, egg and larval mortality rates are generally high, but extremely variable (Ferron \& Legget 1994). This variability is largely attributed to the larva's vulnerability to physical and biological interactions, e.g. predation pressure, starvation and the physical environment (Sissenwine 1984). Such variability contributes significantly to variation in recruitment to the adult population (Narimatsu \& Munehara 1999). Loliginid squid, however, differ fundamentally from fish as they do not exhibit a true larval phase (Young \& Harman 1988). Instead, they undergo direct embryonic development within well-protected, sedentary egg capsules to hatch as behaviourally and structurally adept paralarvae (Boletzky 1987, Boyle et al. 2001). As a consequence, some of the risks associated with a planktonic metamorphosis phase are reduced, resulting in higher survivorship than many marine fishes (Caddy 1983). 
Unfortunately, direct embryonic development in squid is typically a lengthy process, representing up to $30 \%$ of their short lifespan, depending on the species (Boletzky 1987). During this time the developing embryos are potentially at risk to fluctuating environmental conditions (Augustyn et al. 1994). Temperature defines embryonic developmental limits (Segawa 1987) and influence rates of development (Boletzky 1994), and is therefore considered the principal environmental factor governing cephalopod embryonic development. Although some loliginid squids spawn sporadically during the winter months or in deep, cold water (e.g. Loligo forbesi, Lordon \& Casey 1999; Loligo Gahi, Arkhipkin et al. 2000), most species aggregate with some predictability in shallow waters during warmer months to spawn (Hanlon 1998). Spawning during this time and in these regions is assumed to maximise hatching success and survival by effectively accelerating developing embryos through the early vulnerable phase. However, the developing embryos are still potentially vulnerable to rapid temperature and salinity fluctuations, resulting from prevailing weather conditions, while also being at risk of dislodgement due to storm activity (Augustyn et al. 1994, Moltschaniwskyj $\&$ Pecl in press a) and excessive biofouling.

In the laboratory, large fluctuations in temperature and salinity are responsible for major structural deformities and high embryonic mortality (Boletzky \& Hanlon 1983, Palmegiano \& D'Apote 1983, Hanlon 1990, Ueta et al. 1999). In the wild, survivorship may be affected by epiphytic growth on the egg strands' surface, especially for those laid in shallow, nutrient-rich waters and during the later stages of development (Moltschaniwskyj et al. 2002). The effect of epiphytic growth on the developing embryos is unclear, but it may contribute to 'unsatisfactory hatching' (Choe 1966). To date, the relative importance of biotic and abiotic factors on egg development and survival in nature has not been directly assessed due to the difficulties associated with in situ investigations. However, the southern calamary Sepioteuthis australis reliably aggregates in shallow protected waters on the east coast of Tasmania to mate and spawn, allowing researchers regular access to extensive spawning beds. Spawning in $S$. australis is typical of the loliginids; once mated, females attach a series of digitate egg strands to seagrass/macroalgae holdfasts to collectively form discrete egg masses. Individual egg masses may comprise $<10$ to $>500$ strands, with each strand containing between 2 and 8 longitudinally aligned egg capsules (Moltschaniwskyj \& Pecl in press a).

This field-based study aimed to quantify variability in rates of development and mortality for calamary embryos throughout a spring-summer spawning season. Due to the collective egg packaging strategy exhibited by this species, this study also described variation in development within individual egg strands to determine if certain eggs were at a higher risk to mortality. Furthermore, the effect of biofouling on the capsule surface and its effect on mortality rates were quantified.

\section{MATERIALS AND METHODS}

Four southern calamary spawning sites located on the east coast of Tasmania, Australia $\left(42^{\circ} 07^{\prime} 34^{\prime \prime} \mathrm{S}\right.$, $\left.148^{\circ} 17^{\prime} 51^{\prime \prime} \mathrm{E}\right)$ were visited every $2 \mathrm{wk}$ from early November 2000 until early January 2001. All sites were within $5 \mathrm{~km}$ of each other, within $100 \mathrm{~m}$ of the shoreline, less than $4 \mathrm{~m}$ in depth and subjected to a maximum tidal range of $1.2 \mathrm{~m}$. On each trip, divers searched areas of Amphibolis antartica seagrass beds for egg masses containing embryos close to hatching. From each egg mass, 3 biofouled (F) egg strands (75 to $100 \%$ of the surface supporting filamentous algae) and 3 unfouled (UF) egg strands (0\% of the surface supporting filamentous algae) were collected, bagged and stored in fresh seawater prior to dissection.

Embryos were dissected from each strand within $8 \mathrm{~h}$ of collection and examined under a stereo dissection microscope. Developmental stage was assigned to each embryo according to criteria described by Steer et al. (unpubl.) that differ slightly (in terms of the chronological appearance of the eye vesicle, ventral chromatophores, anal papillae and ink sac) from the developmental scheme proposed by Segawa (1987) for Sepioteuthis lessoniana. The position of each embryo within an egg strand was recorded; Position 1 identified the embryo located at the fixed/proximal end of the strand and progressing consecutively to the free/distal end of the strand. Eggs that were unfertilised, had ceased development or were undergoing abnormal development were scored as 'dead'. To avoid over-estimating the within-strand developmental differences, all embryos that had hatched to leave an obviously vacant egg capsule were assumed to have hatched prematurely (Stage 29). This assumption was based on the observation that premature hatching readily occurs during collection (authors' pers obs).

Seawater temperature was measured within seagrass beds using $32 \mathrm{~K}$ StowAway ${ }^{\circledR}$ TidbiT $^{\circledR}$ temperature data loggers (Onset Computer Corp.) located at 3 collection sites. Data loggers were secured to the substrate $(<4 \mathrm{~m}$ deep) 1 mo prior to the first sampling occasion and logged temperature hourly. Daily rainfall data (as a proxy of salinity) were obtained from a local weather station maintained by the Australian Bureau of Meteorology.

To examine conditions experienced by developing embryos sampled on each trip, we back-calculated the 
Table 1. Back-calculated oviposition date for field-collected Sepioteuthis australis eggs using Laptikhovsky's (1999) model. Mean incubation temperature $3 \mathrm{wk}$ prior to collection and developmental stages at time of collection are factored into the equation

\begin{tabular}{|lccccc|}
\hline $\begin{array}{l}\text { Collection } \\
\text { date }\end{array}$ & $\begin{array}{c}\text { Mean } \\
\text { temperature } \\
\left({ }^{\circ} \mathrm{C}\right)\end{array}$ & $\begin{array}{c}\text { Predicted } \\
\text { development time } \\
\text { until hatching }(\mathrm{d})\end{array}$ & $\begin{array}{c}\text { Mean } \\
\text { development } \\
\text { stage }(\mathrm{d})\end{array}$ & $\begin{array}{c}\text { Predicted } \\
\text { development time at } \\
\text { collection (d) }\end{array}$ & $\begin{array}{c}\text { Approx. date } \\
\text { of egg } \\
\text { laying }\end{array}$ \\
\hline 08 Nov 00 & $14.5 \pm 0.04$ & 78.8 & $27.3 \pm 0.10$ & 72.3 & 28 Aug 00 \\
22 Nov 00 & $15.2 \pm 0.05$ & 70.7 & $26.5 \pm 0.08$ & 55.9 & 27 Sep 00 \\
6 Dec 00 & $17.3 \pm 0.04$ & 52.9 & $26.2 \pm 0.09$ & 40.7 & 20 Oct 00 \\
21 Dec 00 & $18.7 \pm 0.05$ & 44.2 & $27.8 \pm 0.12$ & 38.8 & 10 Nov 00 \\
08 Jan 01 & $18.0 \pm 0.07$ & 48.2 & $27.9 \pm 0.08$ & 43.9 & 24 Nov 00 \\
\hline
\end{tabular}

approximate date the eggs were laid. Oviposition dates were calculated using Laptikhovsky's (1999) predictive equation for decapods. This equation takes mean egg size $(\mathrm{L}, \mathrm{mm})$ and incubation temperature $\left(\mathrm{T},{ }^{\circ} \mathrm{C}\right)$ in consideration to generate the duration of embryogenesis ( $D$, days). The equation was applied as

$$
\mathrm{D}=\left(1220.94 \times \mathrm{T}^{-1.68194}\right) \mathrm{L}^{\mathrm{k}}
$$

where $\mathrm{k}=2.5139 \times \mathrm{T}^{-0.3574}$.

Two problems were evident in this equation. Firstly, as the predictive estimates are calculated for eggs developing under constant temperature regimes, they do not account for unknown effects of natural temperature fluctuations observed in the field. Secondly, the equation does not incorporate development stage at the time of collection, but rather calculates the time to hatching. In an attempt to counteract these problems, $\mathrm{T}$ was derived from the average field temperature recorded $3 \mathrm{wk}$ prior to collection, and mean egg development stage at time of collection was factored in to provide approximate oviposition dates (Table 1). L was determined by measuring a total of 375 ovulated eggs collected from the ovaries of 13 mature females (6.18 \pm 0.06, mean $\pm \mathrm{SE}$ ).

To determine variability in embryonic development within egg strands, the average development stage and the deviation of each embryo from the strand average was calculated for all egg positions. If embryonic development was synchronous within an egg strand, each embryo's deviation from the strand mean would be 0 . These differences in developmental deviation within strands were then examined as a function of sampling time, biofouling and position within the strand using a 3-factor Model 1 ANOVA. Only egg strands containing 4 and 5 eggs were included in the analyses to avoid severely unbalancing the data set. All dead embryos were included in the analysis as missing values as developmental stage could not be ascertained. Assumptions of ANOVA were checked by visual inspection of variance and normality plots. No data transformations were required.
To assess whether developmental asymmetry was a function of the number of eggs within a strand, the difference in developmental stage between the fixed and free ends was calculated for all strands containing between 4 and 7 eggs $(n=226)$. Strands containing the extremes (3 and 8 eggs) were not included in the analysis due to insufficient replication. A 1-way ANOVA with unequal replication was used to compare mean values. The Hochberg GT2 post hoc test for an unbalanced data set (Sokal \& Rohlf 2000) was used to highlight significant differences amongst means.

Temporal effects on the frequency of mortality as a function of biofouling and the egg's respective position within the egg strand were estimated via a series of non-parametric log-likelihood ratio $(G)$ tests. Incorporation of Williams's correction $\left(G_{\mathrm{adj}}\right)$ ensured a more conservative estimate of $G$, and therefore reduced the risk of Type I errors (Sokal \& Rohlf 2000).

\section{RESULTS}

Rates of development of Sepioteuthis australis embryos within a strand differed among egg positions, but the pattern and magnitude of the difference along the strand depended upon time $\left(F_{16,601}=3.48, \mathrm{p}=<0.001\right)$. On most occasions, the embryos located at the fixed (proximal) end of the strand developed more slowly than those located at the free (distal) end (Fig. 1). However, in late November, Position 2 embryos were slowest to develop, on average lagging 0.7 developmental stages from the distal embryos (Fig. 1). The withinstrand difference in development was greatest for those eggs collected in early December with proximal embryos on average lagging 1.1 developmental stages behind distal embryos. This difference coincided with the largest incremental change in water temperature, where embryos were subjected to a $7.3^{\circ} \mathrm{C}$ increase over a 41 d developmental period (Fig. 2a). Development rates of embryos within the strand were most synchronous in early November, where proximal and 


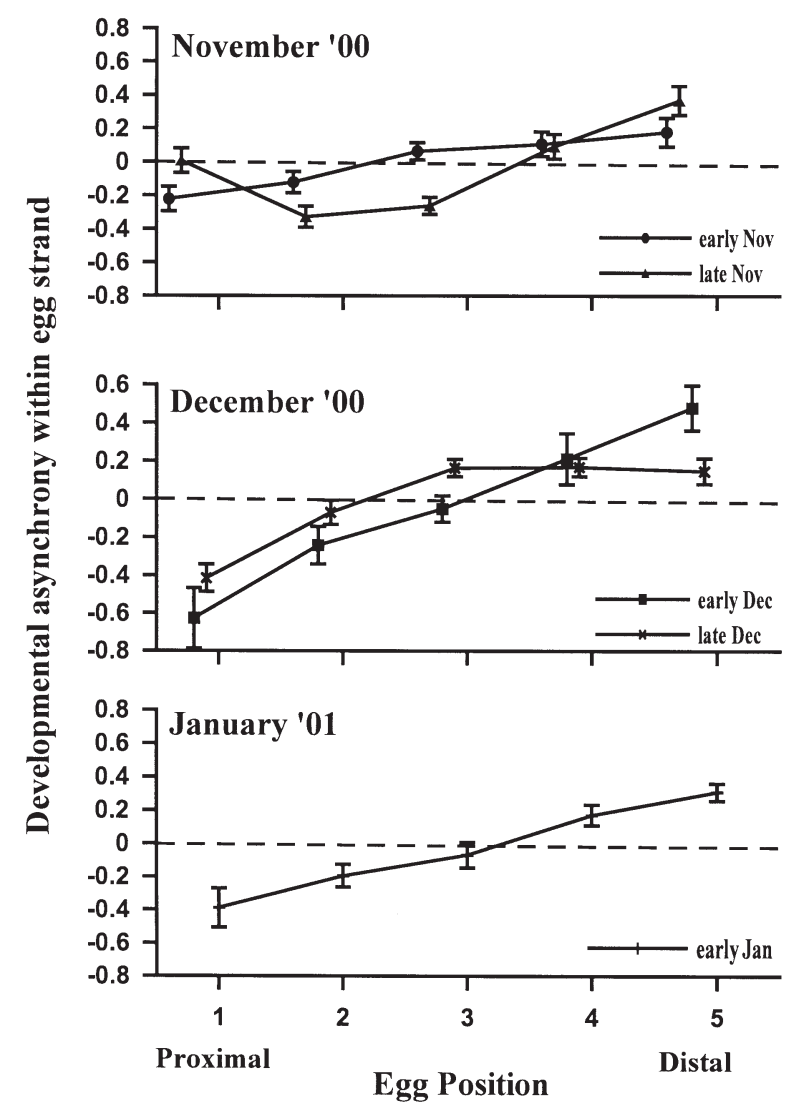

Fig. 1. Sepioteuthis australis. Mean differences in egg development during a spring/summer spawning season. Mean developmental deviation per embryo is calculated as a function of strand mean. Only egg strands containing 4 or 5 egg capsules were included in the analysis $(n=226)$. Dashed line represents synchronous development within an egg strand; error bars represent SE

distal embryos exhibited a mean difference of 0.4 in developmental stage (Fig. 1). Temperatures experienced by developing embryos collected in early November were relatively constant with embryos experiencing a $3.8^{\circ} \mathrm{C}$ range throughout development (Fig. 2a). There was a weak positive correlation between within-strand development variation and rate of temperature change (Pearson's correlation, $r=0.87$, $\mathrm{n}=5, \mathrm{p}=0.05$ ).

Fouling on the egg strands had a large effect on rates of development within the strand with greater withinstrand variation in unfouled than in fouled egg strands $\left(F_{4,601}=13.76, \mathrm{p}=<0.001\right)$. While proximal embryos in unfouled egg strands on average lagged 1.0 development stage behind distal embryos, those in fouled embryos lagged by only 0.4 (Fig. 3).

The number of eggs in the strand affected the difference in the rate of development between the proximal and distal end $\left(F_{3,222}=3.31, \mathrm{p}=0.021\right)$. The shortest egg strands showed minimal difference, with proximal embryos lagging $0.57 \pm 0.17$ developmental stages behind distal embryos. Longer egg strands, however were observed to display greater asynchrony in development with $1.2 \pm 0.2$ developmental stages separating proximal and distal embryos.

Incidence of embryonic mortality changed over the summer, ranging from $\sim 4 \%$ dead in early November to $19.5 \%$ in late November (Fig. 4). Total mortality remained above $10.0 \%$ in early December and January, periodically dropping to $6.4 \%$ in late December, and greatly varied as a function of biofouling $\left(G_{\mathrm{adj}}=\right.$ $<89.27, \mathrm{p}=<0.001$ ). From late November onwards, the incidence of mortality in unfouled egg strands was as much as $90 \%$ higher than that in fouled strands. Except in early November, mortality rates were very similar in both fouled and unfouled egg strands.

Using Laptikhovsky's (1999) predictive equation, it was possible to back-calculate the approximate oviposited dates from sampled embryos. Embryos collected in the initial sample were back-calculated to

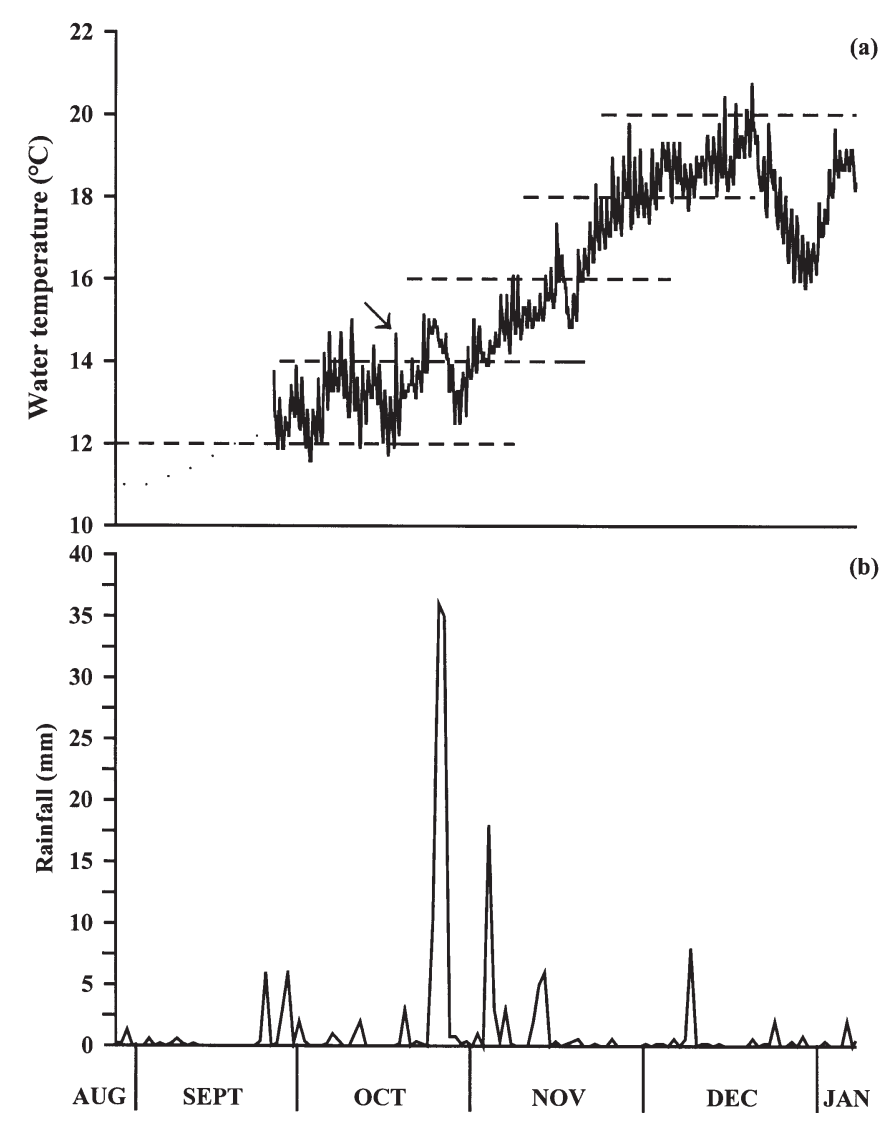

Fig. 2. Sepioteuthis australis. (a) Benthic water temperature $\left({ }^{\circ} \mathrm{C}\right)$ measured hourly; dashed horizontal lines represent duration of embryonic development of collected egg samples calculated using Laptikhovsky's (1999) predictive equation for decapods. Arrow indicates the greatest temperature spike observed during the study period. (b) Daily rainfall (mm) 


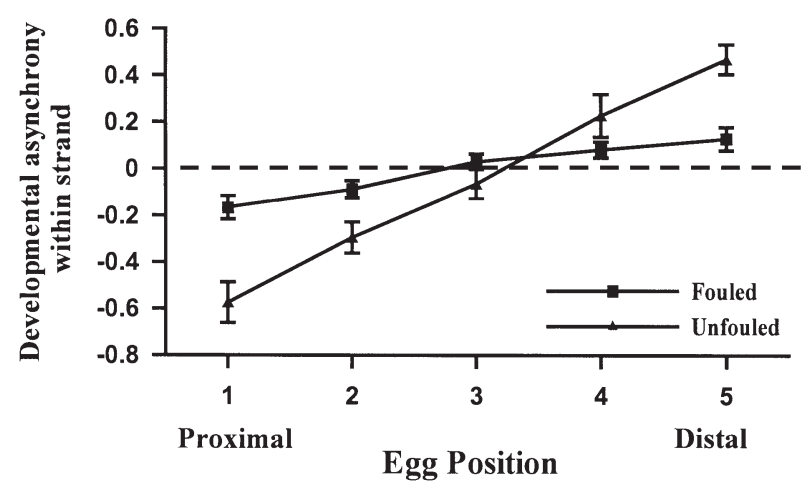

Fig. 3. Sepioteuthis australis. Mean differences in egg development between fouled $(\mathrm{n}=350)$ and unfouled strands $(\mathrm{n}=$ 301). Mean developmental deviation per embryo is calculated as a function of strand mean. Fouled $=75$ to $100 \%$ surface coverage; unfouled $=0 \%$ surface coverage. Dashed line represents synchronous development within an egg strand; error bars represent SE

have been laid on 28 August, marking the 'beginning' of the study period (Table 1). By calculating the oviposited date, it is possible to identify potential environmental perturbations throughout embryonic development, i.e. from when eggs were laid to when they were sampled (Fig. 2a). Diel temperature fluctuations were small throughout the study $\left(1.18 \pm 0.55^{\circ} \mathrm{C}\right.$, mean $\pm \mathrm{SD}$ ). The largest temperature change occurred on 17 October, when there was an increase of $2.8^{\circ} \mathrm{C}$ over a $7 \mathrm{~h}$ period (Fig. 2a). All embryos collected in November would have experienced this subtle temperature spike during their developmental process; however, there was no correlation with increased mortality rates and changes in water temperature throughout the study (Pearson's correlation, $\mathrm{r}=0.19, \mathrm{n}=5, \mathrm{p}=0.49$; Fig. 2a). Embryos collected in November and early

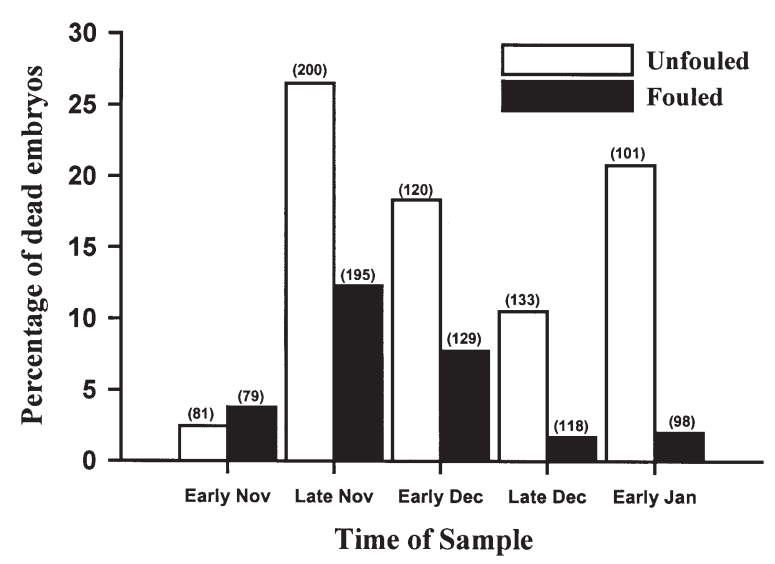

Fig. 4. Sepioteuthis australis. Percentage frequency of dead embryos at each sampling time across all strand positions for fouled and unfouled egg strands. Numbers in parentheses indicate total number of embryos examined

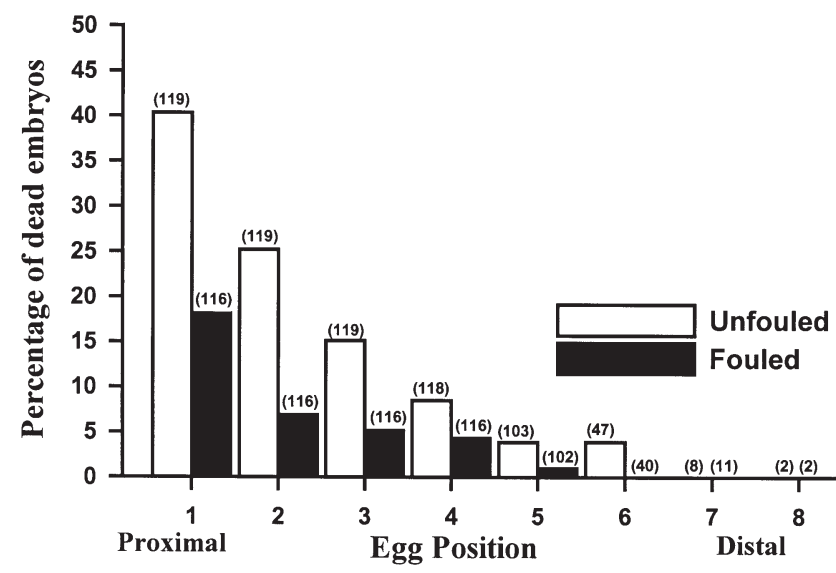

Fig. 5. Sepioteuthis australis. Percentage frequency of dead embryos at each position along the egg strand for fouled and unfouled egg strands. Numbers in parentheses indicate total number of embryos examined

December experienced a period of heavy rainfall where a total of $83.2 \mathrm{~mm}$ fell over $7 \mathrm{~d}$ from 24 to 30 October, with major downpours occurring on the 25th and 26th measuring 36 and $35 \mathrm{~mm}$, respectively. (Fig. 2b). Rainfall during this period considerably exceeds the October average of $50 \mathrm{~mm}$ (Australian Bureau of Meteorology, www.bom.gov.au).

Embryos developing at the proximal end of an egg strand suffered mortality rates more than 7 times higher than those at the distal end; a pattern that was consistent in both fouled and unfouled strands $\left(G_{\text {adj }}=147.74\right.$, $\mathrm{p}=<0.001$; Fig. 5). No dead embryos were found in Positions 7 or 8 regardless of biofouling (Fig. 5).

\section{DISCUSSION}

Southern calamary embryos develop asynchronously within individual egg strands with proximal embryos consistently developing slower and suffering higher mortality than their distal siblings. Variable development within an aggregated egg mass has been reported in a variety of marine species, e.g. gastropods Lymnaea stagnalis, Melanochlamys diomedeai and Haminaea vesicula, polychaete Nereis vexillosa (Marois \& Croll 1991, Cohen \& Strathmann 1996) and fish Clupea harengus (Stratoudakis et al. 1998). In each instance, embryos in the distal or outer perimeter of the egg mass develop faster and hatch first. This difference in developmental rate is attributed to the interior embryos not getting sufficiently oxygenated and/ or accumulating excess excretory products, and responding by either retarding or arresting development (Strathmann \& Strathmann 1995). Given the high density of eggs in the squid egg masses, particularly at the 
attachment point, it would be of value to determine the micro-environmental characteristics experienced by the embryos.

Temporal variations in developmental rate and mortality estimates were explored as a function of incubation temperature. The positive correlation between the magnitude of within-strand developmental variation and incubation temperature range suggests that asynchronous development was greater when embryos were subjected to a wider temperature range. Importantly, this can translate to differential hatch times, suggesting that an entire egg mass will hatch over a longer period when incubation temperatures change, compared to egg masses experiencing relatively stable temperatures. Similar results have been observed in shallow-water, benthic spawning fish, such as Pacific herring Clupea pallasii and capelin Mallotus villosus, where the hatching interval was negatively correlated with the average incubation temperature (Taylor 1971, Frank \& Leggett 1981). Given that temperature intrinsically defines developmental rates, it is likely that when embryos are subjected to progressively warmer temperatures, typical of a seasonal increase, embryonic development speeds up, exaggerating the withinstrand variation. It is unclear as to whether survival of remaining embryos is compromised once hatching begins.

Although seasonal increases in temperature are positively correlated with developmental rate, their influence on embryonic mortality remains unclear. Temporal differences in embryonic mortality have been observed in the long-finned squid Loligo gahi, where the incidence of mortality during the winter months is more than 4 times greater than during spring and summer (Arkhipkin et al. 2000). It remains to be investigated whether this seasonal difference was purely a result of differing temperatures, or other environmental conditions, or a combination of both. Extreme temperature fluctuations are suggested to be detrimental in laboratory-reared eggs, especially during the earlier developmental stages, and controlled temperature shifts are advised not to exceed $1^{\circ} \mathrm{C} \mathrm{d}^{-1}$ (Hanlon 1990). As temperature fluctuations during this study were generally between 1 and $2^{\circ} \mathrm{C} \mathrm{d}^{-1}$, it is unlikely that thermal conditions contributed to elevated mortality. A previous study of late-stage Sepioteuthis lessoniana embryos showed that short-term exposure $(<1 \mathrm{~h})$ to temperatures 3 to $7^{\circ} \mathrm{C}$ above natural conditions does not adversely affect development (Kinoshita 1982). As the maximum rate of temperature elevation recorded in this study equated to $2.8^{\circ} \mathrm{C}$ over a $7 \mathrm{~h}$ period, it is unlikely to have promoted developmental arrest. A study by Pedersen \& Tande (1992) suggests that invertebrates living in environments characterised by natural increases in temperature during the developmen- tal period are physiologically adapted to cope with slight fluctuations. By this rationale, Sepioteuthis australis embryos may be relatively robust with regard to coping with temperature fluctuations.

Heavy rainfall and subsequent freshwater run-off in October may have contributed to elevated mortalities observed in late November and early December. As all eggs examined in this study were collected in shallow, nearshore waters $(<4 \mathrm{~m})$, their development may have been perturbed by rapid changes in salinity. Cephalopods are generally thought to be stenohaline, and supporting field evidence suggest that low salinities have a significant inhibitory effect on hatching success in the cuttlefish Sepia officinalis (Palmegiano \& D'Apote 1983). Embryos that were collected in early November would have experienced this change in salinity; however, they displayed a relatively low incidence of embryonic mortality, suggesting that if salinity was to have an effect on development, the timing of such events may prove to be an important factor.

Biofouling did not seem to have any obvious detrimental effect upon embryonic development. Embryos developing within fouled strands displayed relative synchrony in development and a low incidence of mortality compared to those developing within unfouled strands. This result was unexpected, as epiphytic growth upon amphibian eggs typically creates hypoxic conditions during the night (Pinder \& Friet 1994). It has been suggested that colonisation of the fouling organisms on Sepioteuthis australis egg strands is delayed by a chemical defence present on the strand's surface. Therefore, any resultant growth is not rapid enough to interfere with development, as embryos hatch in sufficient time before fouling has any effect (Benkendorff 1999). Conversely, the photosynthetic abilities of fouling organisms coupled with the surrounding Amphibolis vegetation may enhance oxygen levels during the day, and water movement during the night may cancel out any negative effect of epiphyte respiration (Cohen \& Strathmann 1996). Excessive biofouling may additionally benefit developing embryos by protecting them from potentially damaging solar radiation that has been known to cause problems in development in other taxa (Biermann et al. 1992), and perhaps even explain why mortality was higher in strands that were free of fouling.

The present study demonstrates temporal variability in development rates of Sepioteuthis australis embryos. The dynamic nature of shallow-water spawning sites makes it difficult to single out the major contributing factor(s) responsible for embryonic mortality. As a result, future work should focus upon defining tolerance levels in developing embryos exposed to a variety of fluctuating environmental conditions, and determine their relative effect on different phases of 
development. In addition, the size and density of an egg mass needs to be factored into any analysis to determine if this is contributing to early mortality rates (see Strathmann \& Strathmann 1995).

Commercial catches of Sepioteuthis australis off Tasmania have increased in recent years and a series of fishery closures aimed at protecting the spawning stock and maximising recruitment strength were implemented (Moltschaniwskyj et al. in press b). Understanding the effect of the environment on embryonic development and hatching success can reduce some of the variability encompassed within existing stockrecruitment relationships, which are currently based on spawner biomass/parental stock sizes. Reducing variability within predictive stock-recruitment relationships will allow fisheries managers to make more informed and accurate decisions about the fishery.

Acknowledgements. Thanks to G. Pecl, S. Willcox and S. Talbot for valuable field assistance, and A. Jordan and B. McGrath for their critical review of earlier drafts. The authors also wish to thank E. G. Dawe and an anonymous reviewer for constructive comments made to the manuscript. M.A.S. was supported by a Tasmania Research Scholarship with supplementary funding from the Tasmanian Aquaculture and Fisheries Institute. F.C.G. was supported by a travel grant from The British Society of Developmental Biology. All field expenses were met by a concurrent project funded by FRDC (Fisheries Research and Development Corporation) (2000/121) and awarded to N.A.M.

\section{LITERATURE CITED}

Arkhipkin AI, Laptikhovsky VV, Middleton DAJ (2000) Adaptions for cold water spawning in loliginid squid: Loligo gahi in Falkland waters. J Molluscan Stud 66:551-564

Augustyn CJ, Lipinski MR, Sauer WHH, Roberts MJ, Mitchell-Innes BA (1994) Chokka squid on the Agulhas Bank: life history and ecology. S Afr Tydskr Wet 90: 143-153

Benkendorff K (1999) Bioactive molluscan resources and their conservation: biological and chemical studies on the egg masses of marine molluscs. PhD thesis, University of Wollongong, NSW

Biermann CH, Schinner GO, Strathmann RR (1992) Influence of solar radiation, microalgal fouling, and current on deposition site and survival of embryos of a dorid nudibranch gastropod. Mar Ecol Prog Ser 86(3):205-215

Boletzky SV (1987) Embryonic phase. In: Boyle PR (ed) Cephalopod life cycles, Vol II. Academic Press, London, p 5-31

Boletzky SV (1994) Embryonic development of cephalopods at low temperatures. Antarct Sci 6(2):139-142

Boletzky SV, Hanlon RT (1983) A review of the laboratory maintenance, rearing and culture of cephalopod molluscs. Mem Natl Mus Vic 44:147-187

Boyle PR, Noble L, Emery AM, Craig S, Black KD, Overnall J (2001) Development and hatching in cephalopod eggs: a model system for partitioning environmental and genetic effects on development. In: Atkinson D, Thorndyke M (eds) Environment and animal development: genes, life history and plasticity. BIOSIS Scientific Publishers, Oxford, p 251-267

Caddy JF (1983) The cephalopods: factors relevant to their population dynamics and to the assessment and management of stocks. In: Caddy JF (ed) Advances in assessment of world cephalopod resources. FAO Fish Tech Pap 231: $416-449$

Choe S (1966) On the eggs, rearing, habits of the fry, and growth of some cephalopoda. Bull Mar Sci 16:330-348

Cohen CS, Strathmann RR (1996) Embryos at the edge of tolerance: effects of environment and structure of egg masses on supply of oxygen to embryos. Biol Bull 190:8-15

Ferron A, Leggett WC (1994) An appraisal of condition measures for marine fish larvae. Adv Mar Biol 30:217-303

Frank KT, Leggett WC (1981) Prediction of egg development and mortality rates in capelin (Mallotus villosus) from meteorological, hydrodynamic and biological factors. Can J Fish Aquat Sci 38:1327-1338

Hanlon RT (1990) Maintenance, rearing and culture of teuthoid and sepioid squids. Plenum Press, New York

Hanlon RT (1998) Mating systems and sexual selection in the squid Loligo: how might commercial fishing on spawning grounds affect them? CalCOFI Rep 39:92-99

Kinoshita H (1982) Thermal tolerance of eggs and larvae of a squid Sepioteuthis lessoniana Lesson, (Decapoda, Mollusca). Rep Mar Ecol Res Inst 82201:1-30

Laptikhovsky VV (1999) Improved mathematical model to study the duration of embryogenesis in cephalopod molluscs. Ruthenica 9(2):141-146

Lordan C, Casey J (1999) The first evidence of offshore spawning in the squid species Loligo forbesi. J Mar Biol Assoc UK 79:379-381

Marois R, Croll RP (1991) Hatching asynchrony within the egg mass of the pond snail, Lymnaea stagnalis. Invertebr Reprod Dev 19(2):139-146

Moltschaniwskyj NA, Pecl G (in press a) Small-scale spatial and temporal patterns of egg production by the temperate loliginid squid Sepioteuthis australis. Mar Biol

Moltschaniwskyj N, Pecl G, Lyle J (in press b) An assessment of the use of short-term closures to protect spawning southern calamary aggregations from fishing pressure in Tasmania, Australia. Bull Mar Sci

Narimatsu Y, Munehara H (1999) Spawn date dependent survival and growth in the early life stages of Hypoptychus dybowskii (Gasterosteiformes). Can J Fish Aquat Sci 56: 1849-1855

O'Dor RK (1998) Can understanding squid life-history strategies and recruitment improve management? S Afr J Mar Sci 20:193-206

Palmegiano GB, D'Apote MP (1983) Combined effects of temperature and salinity on cuttlefish (Sepia officinalis L.) hatching. Aquaculture 35:259-264

Pedersen G, Tande KS (1992) Physiological plasticity to temperature in Calanus finmarchicus: reality or artefact? J Exp Mar Biol Ecol 155(2):183-197

Pinder AW, Friet SC (1994) Oxygen transport in egg masses of the amphibians Rana sylvatica and Ambrystoma maculatum-convection, diffusion and oxygen production by algae. J Exp Biol 197:17-30

Segawa S (1987) Life history of the oval squid, Sepioteuthis lessoniana in Kominato and adjacent waters Central Honshu, Japan. J Tokyo Univ Fish 74(2):67-105

Sissenwine JP (1984) Why do fish populations vary? In: May R (ed) Exploration of marine communities. Springer-Verlag, Berlin, p 59-94

Sokal RR, Rohlf FJ (2000) Biometry. WH Freeman, New York Strathmann RR, Strathmann MF (1995) Oxygen supply and 
limits on aggregation of embryos. J Mar Biol Assoc UK 75: 413-428

Stratoudakis Y, Gallego AG, Morrison JA (1998) Spatial distribution of developmental egg ages within a herring $\mathrm{Clu}$ pea harengus spawning ground. Mar Ecol Prog Ser 174: $27-32$

Taylor FHC (1971) Variation in hatching success in Pacific herring (Clupea pallasii) eggs with water depth, tempera-

Editorial responsibility: Otto Kinne (Editor),

Oldendorf/Luhe, Germany ture, salinity and egg mass thickness. Rapp P-V Reun Cons Int Explor Mer 160:34-41

Ueta Y, Tokai T, Segawa S (1999) Relationship between yearclass abundance of the oval squid Sepioteuthis lessoniana and environmental factors off Tokushima Prefecture, Japan. Fish Sci 65:424-431

Young RE, Harman RF (1988) 'Larva,' 'paralarva' and 'subadult' in cephalopod terminology. Malacologia 29:201-207

Submitted: April 2, 2002; Accepted: August 26, 2002

Proofs received from author(s): October 31, 2002 